\title{
Stress of Vertical Cylindrical Vessel for Thermal Stratification of Contained Fluid
}

\author{
Ichiro Furuhashi \\ Mito Science Analysis Intelligence Corp. Mito Ibaraki \\ Japan
}

\section{Introduction}

Various thermal loads are induced in elevated temperature systems, such as nuclear power plants. The load caused by the thermal stratification of contained fluid is one of those loads (Moriya et al., 1987; Bieniussa \& Reck, 1996; Kimura et al., 2010). The thermal stratification is phenomenon under the condition of insufficient forced-convection mixture, where a denser fluid layer of lower temperature locates beneath a lighter fluid layer of higher temperature (Haifeng et al., 2009).

A conventional design evaluation method of vessel stress assumes an axial vessel temperature profile consisting of a straight line with the maximum fluid temperature gradient as shown in the top of Fig.1, and applies cylindrical shell theory for stress solution (Timoshenko \& Woinowsky, 1959). The conventional method gives conservative solutions of thermal stresses that are proportional to the temperature gradient, and hence leads to narrower design windows.

In actual conditions, thermal stress is smaller than that from the conventional method, because of relatively moderated temperature profile due to attenuation by heat transfer on the inner surfaces and by heat conduction in vessel walls as shown in the bottom of Fig.1, as well as the cancellation of stresses at both ends of the thermal stratification section that have opposite signs generated by the reverse temperature changes. The consideration of such effects conventionally requires FEM heat conduction analyses taking the heat transfer with fluid into account and the subsequent FEM thermal stress analyses based on the above results. However, the FEM analyses are not suitable for a design work which places a high priority to get design perspective with rapid estimation.

In order to propose an accurate design method, this paper studies the steady-state vessel temperature solutions based on a model shown in Fig.2 taking the heat transfer with fluid and heat conduction into account, as well as the subsequent cylindrical shell stress ones based on the above temperature results. The obtained results are compiled into easy-to-use charts for design.

\section{Theoretical analysis}

The analysis model is shown in Fig.2. It is assumed that the radius of a cylindrical vessel, $R$, is enough larger than the vessel thickness, $t(R>>t)$, so that the vessel wall can be considered as a flat plate. Here, $\lambda$ is the thermal conductivity of the vessel. The positions in the plate thickness direction and the axial direction are represented by $x$ and $z$, respectively. 

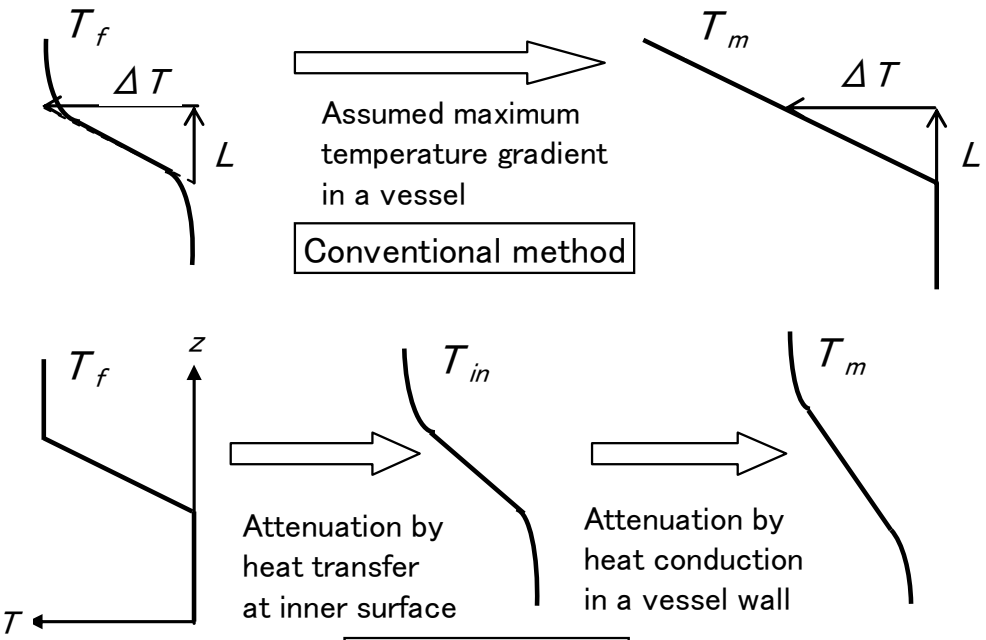

Proposed method

Fig. 1. Comparison of conventional and proposed methods

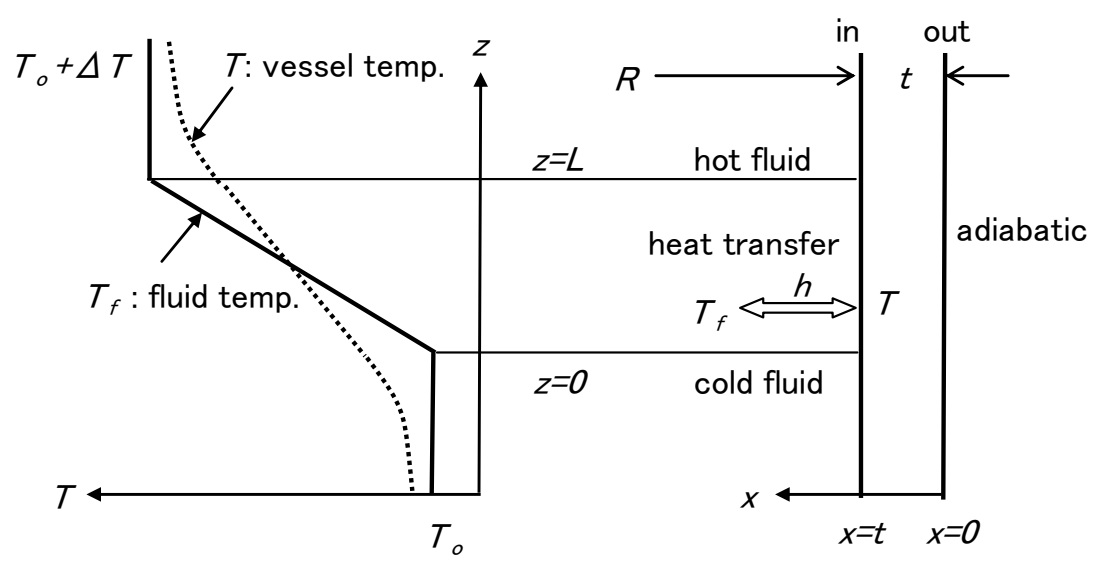

Fig. 2. Analysis model

It is assumed that the external surface of the vessel $(x=0)$ is thermally-insulated and heat transfer occurs between the inner surface $(x=t)$ and fluid with the heat transfer coefficient, $h$. The thermal stratification layer is represented by the range of $z=0 \sim L$.

\subsection{Step-shaped fluid temperature profile}

We discuss the case in which the fluid temperature profile is given by the following step function (in the case of $L=0$ in Fig.2).

$$
T_{f}(z)=T_{0}+H(z) \Delta T
$$


Here, $H(z)$ is a step function; $H(z)=0$ for $z<0$, and $H(z)=1$ for $z>0$. Using the eigen-function expansion method (Carslaw \& Jeager, 1959), the steady-state vessel wall temperature, $T(x, z)$, was obtained as the following equation (Furuhashi et al., 2007, 2008).

$$
T(x, z)=T_{0}+H(z) \Delta T-\operatorname{sgn}(z) \sum_{n=1}^{\infty} c_{n} \cos \left(p_{n} x\right) \exp \left(-p_{n}|z|\right)
$$

Here, $\operatorname{sgn}(z)$ is a sign function; $\operatorname{sgn}(z)=-1$ for $z<0$, and $\operatorname{sgn}(z)=1$ for $z>0$. Each term of the series is an eigen-function that satisfies the steady-state condition, $\partial^{2} T / \partial x^{2}+\partial^{2} T / \partial z^{2}=0$, and the adiabatic condition at $x=0$ plane, $\partial T / \partial x=0$. The eigen-values, $p_{n}(n=1,2, \ldots)$, consist of positive roots in ascending order of eigen-value equation, Eq.(3), that is derived from the heat transfer condition at $x=t$ plane, $\lambda \partial T / \partial x=h\left(T_{f}-T\right)$.

$$
\left(p_{n} t\right) \tan \left(p_{n} t\right)=\frac{h t}{\lambda}=B i
$$

Here, $B i$ is the non-dimensional heat transfer coefficient (Biot number). The coefficients, $c_{n}$ $(n=1,2, \ldots)$, are obtained from the symmetry condition, $T(x,+0)=T(x,-0)=T_{0}+\Delta T / 2$.

$$
c_{n}=\frac{\Delta T \sin \left(p_{n} t\right)}{p_{n} t+\sin \left(p_{n} t\right) \cos \left(p_{n} t\right)}
$$

The wall-averaged temperature is represented by the following equation.

$$
T_{m}(z)=\frac{1}{t} \int_{0}^{t} T(x, z) d x=T_{0}+H(z) \Delta T-\operatorname{sgn}(z) \sum_{n=1}^{\infty} \frac{c_{n}}{p_{n} t} \sin \left(p_{n} t\right) \exp \left(-p_{n}|z|\right)
$$

The values calculated by the theoretical solution of wall-averaged temperature, Eq. (5), for $B i=0.1,1,10,100$ are plotted in Fig. 3 with the note, (exact), in the legend.

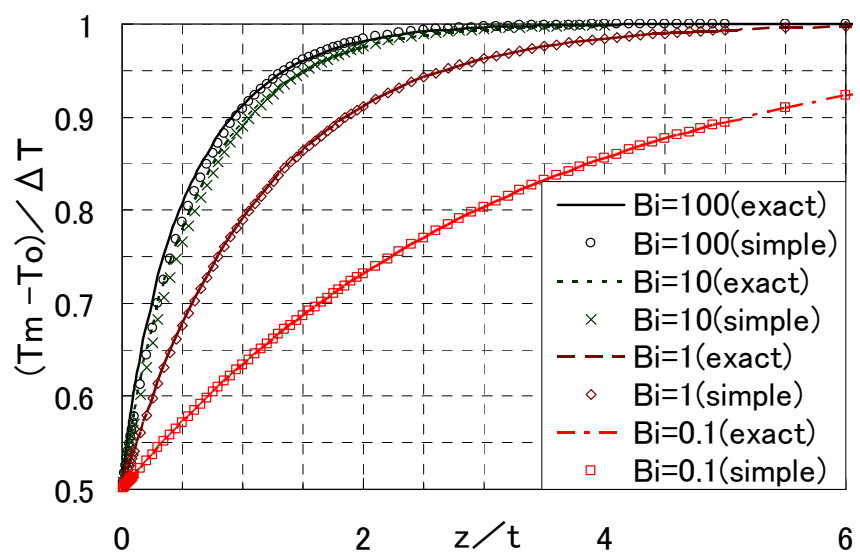

Fig. 3. Comparisons of vessel temperatures by exact solution with those by the temperature profile method 


\subsection{Simplified solution based on the temperature profile method}

The theoretical solution, Eq.(5), is convenient for the calculation on a PC and quite useful. However, it is not a simplified equation suitable for the design evaluation because it needs a series calculation and an eigen-value calculation. Then, we tried to obtain an approximate simple solution that allows easy calculation based on the temperature profile method (Katto, 1964). The axial profile of wall-averaged temperature is approximated by the following equation (Furuhashi et al., 2007, 2008).

$$
T_{m}(z)=T_{0}+H(z) \Delta T-\operatorname{sgn}(z) \frac{\Delta T}{2} e^{-b|z|}
$$

Here, the constant $b$ is termed as temperature attenuation coefficient. When assuming that the temperature profile in the plate thickness direction is parabolic; $T=a_{0}(z)+a_{1}(z) x^{2}$, then next equations holds.

$$
\begin{gathered}
T_{\text {in }}=a_{0}(z)+a_{1}(z) t^{2} \\
T_{m}=\int_{0}^{t} T d x / t=a_{0}(z)+a_{1}(z) t^{2} / 3
\end{gathered}
$$

Here, $T_{\text {in }}$ represents the inner surface temperature. Temperature gradient at the inner surface in the thickness direction is given by the following equation.

$$
\left.\frac{\partial T}{\partial x}\right|_{x=t}=2 a_{1}(z) t=\frac{3}{t}\left(T_{i n}-T_{m}\right)
$$

Using this equation, heat flux at the vessel inner surface, $q$, is given approximately by the following equation.

$$
q=h\left(T_{f}-T_{i n}\right)=\left.\lambda \frac{\partial T}{\partial x}\right|_{x=t}=\frac{3 h}{3+B i}\left(T_{f}-T_{m}\right)
$$

Then the total heat flow from fluid to the vessel in the hot side $(z>0), Q$, is given approximately by the following equation.

$$
Q=2 \pi R h \int_{0}^{\infty}\left(T_{f}-T_{i n}\right) d z=\frac{6 \pi R h}{(3+B i)} \int_{0}^{\infty}\left(T_{f}-T_{m}\right) d z=\frac{3 \pi R h \Delta T}{(3+B i) b}
$$

The heat flow from the hot side to the cold side across the $z=0$ plane, $Q$, is given by the following equation.

$$
Q=\left.2 \pi R t \lambda \frac{d T_{m}}{d z}\right|_{z=0}=\pi R t \lambda \Delta T b
$$

Since Eqs.(11) and (12) are equivalent in the steady-state, then the coefficient $b$ can be obtained by the following equation (Furuhashi et al., 2007, 2008).

$$
b=\frac{1}{t} \sqrt{\frac{3 B i}{3+B i}}
$$


The values calculated by the approximate solution of wall-averaged temperature, Eq.(6), for $B i=0.1,1,10,100$ are plotted in Fig. 3 with the note, (simple), in the legend. The maximum relative error to the theoretical solution, Eq.(5), is $0.01 \%$ for $B i=0.1,0.3 \%$ for $B i=1,2.0 \%$ for $B i=10$ and $3.3 \%$ for $B i=100$. Consequently, a high-precision easy-to-use approximate solution is obtained.

\subsection{Cylindrical shell solution of steady-state thermal stress}

Young's modulus, thermal expansion coefficient, and Poisson's ratio of the vessel is represented by $E$, $a$, and $v$, respectively. When the vessel wall is in the context of mechanical free boundary conditions, the radial outward displacement, $u(z)$, can be obtained as the solution of the following differential equation (Timoshenko \& Woinowsky, 1959; Furuhashi \& Watashi, 1991).

$$
\frac{d^{4} u}{d z^{4}}+4 \beta^{4} u=\frac{p(z)}{D}+\frac{E t \alpha}{D R} T_{m}(z)+\frac{(1+v) \alpha}{t} \frac{d^{2} T_{b}(z)}{d z^{2}}
$$

Here, $p(z)$ is the inner pressure, and $p(z)=0$ is assumed. $T_{b}(z)$ is the equivalent linear temperature difference, representing the "inner surface temperature minus outer surface temperature" in the case that the temperature profile in the wall thickness direction is linearly approximated. Here, $T_{b}(z)=0$ is assumed. The acceptability of this assumption will be checked in the comparisons made after. $D$ is the flexural rigidity of the wall, and $\beta$ is the stress decay coefficient.

$$
\begin{gathered}
D=\frac{E t^{3}}{12\left(1-v^{2}\right)} \\
\beta=\sqrt[4]{\frac{E t}{4 D R^{2}}}=\frac{\sqrt[4]{3\left(1-v^{2}\right)}}{\sqrt{R t}}
\end{gathered}
$$

The axial bending stress $\sigma_{z b}$, circumferential membrane stress $\sigma_{h m}$ and circumferential bending stress $\sigma_{h b}$ is given by the following equations, respectively (Timoshenko \& Woinowsky, 1959; Furuhashi \& Watashi, 1991).

$$
\begin{gathered}
\sigma_{z b}(z)=\frac{6 D}{t^{2}}\left\{\frac{d^{2} u}{d z^{2}}-\frac{(1+v) \alpha T_{b}}{t}\right\} \\
\sigma_{h m}(z)=E\left\{\frac{u}{R}-\alpha T_{m}\right\} \\
\sigma_{h b}(z)=\frac{6 D}{t^{2}}\left\{v \frac{d^{2} u}{d z^{2}}-\frac{(1+v) \alpha T_{b}}{t}\right\}
\end{gathered}
$$

The radial displacement was solved as the following equation by substituting the approximate solution of $T_{m}(z)$, Eq.(6), into the right side of Eq.(14) (Furuhashi et al., 2007, 2008).

$$
u(z)=R \alpha T_{m}(z)+\operatorname{sgn}(z) \frac{m R \alpha \Delta T}{2} e^{-b|z|}-\operatorname{sgn}(z) \frac{m R \alpha \Delta T}{2} e^{-\beta|z|} \cos (\beta z)-n R \alpha \Delta T e^{-\beta|z|} \sin (\beta z)
$$


Here, parameters, $m$ and $n$, is given by the following equations, respectively.

$$
\begin{aligned}
& m=\frac{b^{4}}{4 \beta^{4}+b^{4}}=\frac{(b / \beta)^{4}}{4+(b / \beta)^{4}} \\
& n=\frac{\beta^{2} b^{2}}{4 \beta^{4}+b^{4}}=\frac{(b / \beta)^{2}}{4+(b / \beta)^{4}}
\end{aligned}
$$

The thermal stresses were solved as the following equations by substituting Eq.(20) into Eqs.(17), (18) and (19) (Furuhashi et al., 2007, 2008).

$$
\begin{gathered}
S_{z b}(z)=\frac{\sigma_{z b}(z)}{E \alpha \Delta T}=\frac{\sqrt{3}}{\sqrt{1-v^{2}}}\left\{-\operatorname{sgn}(z) n \cdot e^{-b|z|}-\frac{m}{2} e^{-\beta|z|} \sin (\beta z)+\operatorname{sgn}(z) n \cdot e^{-\beta|z|} \cos (\beta z)\right\} \\
S_{h m}(z)=\frac{\sigma_{h m}(z)}{E \alpha \Delta T}=\operatorname{sgn}(z) \frac{m}{2} e^{-b|z|}-\operatorname{sgn}(z) \frac{m}{2} e^{-\beta|z|} \cos (\beta z)-n \cdot e^{-\beta|z|} \sin (\beta z) \\
S_{h b}(z)=\frac{\sigma_{h b}(z)}{E \alpha \Delta T}=v S_{z b}(z)
\end{gathered}
$$

Here, $S$ represents the non-dimensional stress normalized by $E a \Delta T$. These equations suggest that the non-dimensional stress profiles, the plots of $S$ with regard to $\beta z$, exclusively depend on the ratio of coefficients, $b / \beta$, because both $m$ and $n$ are functions of $b / \beta$ only.

When $t$ approaches $0(t=>0), b / \beta=>\infty, m=>1, n=>0$ and $T_{m}(z)=>T_{f}(z)$, and consequently, thermal stresses approach the following equations.

$$
\begin{aligned}
& S_{z b}(z) \rightarrow-\frac{\sqrt{3}}{2 \sqrt{1-v^{2}}} e^{-\beta|z|} \sin (\beta z) \\
& S_{h m}(z) \rightarrow-\frac{\operatorname{sgn}(z)}{2} e^{-\beta|z|} \cos (\beta z)
\end{aligned}
$$

Those limit solutions for $b / \beta=>\infty$ yield the maximum at the $z$ values shown in the following equations, lead to the upper limit of thermal stresses for free boundary conditions.

$$
\begin{gathered}
S_{z b, \text { Lim }}\left(z= \pm \frac{\pi}{4 \beta}\right)=\mp 0.293(v=0.3) \\
S_{h m, \text { Lim }}(z= \pm 0)=\mp 0.5
\end{gathered}
$$

\subsection{Ramp-shaped fluid temperature profile}

The Green functions (indicial response functions) are obtained from the temperature, displacement and stress of the vessel based on the step-shaped fluid temperature profile (Carslaw \& Jeager, 1959; Morse \& Feshbach, 1953). When any-shaped fluid temperature profile in z-axis direction is given, the temperature, displacement and stress of the vessel in steady-state can be obtained by convolution integral. We discuss the case in which a ramp- 
shaped fluid temperature profile is given as shown in Fig.2. This profile is expressed by the following equations.

$$
\begin{aligned}
& T_{f}(z)=T_{0} \quad(z<0) \\
& T_{f}(z)=T_{0}+\Delta T \frac{z}{L} \quad(0 \leq z \leq L) \\
& T_{f}(z)=T_{0}+\Delta T \quad(z>L)
\end{aligned}
$$

The $G_{T}(z)$ that are obtained from Eq.(6) with $T_{0}=0$ and $\Delta T=1$ express the approximate Green function of wall-averaged temperature of the vessel when the fluid temperature profile is given by a unit step function.

$$
\begin{aligned}
& G_{T}(z)=\frac{1}{2} e^{b z} \quad(z<0) \\
& G_{T}(z)=1-\frac{1}{2} e^{-b z} \quad(0 \leq z)
\end{aligned}
$$

When the fluid temperature profile is given by a ramp-shaped function expressed by Eq.(30), the wall-averaged temperature of the vessel was obtained by convolution integral as the following equation (Furuhashi et al., 2007, 2008).

$$
\begin{aligned}
& T_{m}(z)=T_{f}(-\infty)+\int_{-\infty}^{\infty} \frac{d T_{f}(\xi)}{d \xi} G_{T}(z-\xi) d \xi=T_{0}+\frac{\Delta T}{L} \int_{0}^{L} G_{T}(z-\xi) d \xi \\
& =T_{f}(z)+\frac{\Delta T}{2 b L}\left(e^{-b|z|}-e^{-b|z-L|}\right)
\end{aligned}
$$

Similarly, the $G_{u}(z)$ that are obtained from Eq.(20) with $T_{0}=0$ and $\Delta T=1$ express the approximate Green function of radial displacement of the vessel when the fluid temperature profile is given by a unit step function.

$$
\begin{aligned}
& G_{u}(z)=\operatorname{R} \alpha\left\{H(z)-\operatorname{sgn}(z) \frac{1}{2} e^{-b|z|}\right\}+\operatorname{sgn}(z) \frac{m R \alpha}{2} e^{-b|z|} \\
& -\operatorname{sgn}(z) \frac{m R \alpha}{2} e^{-\beta|z|} \cos (\beta z)-n R \alpha e^{-\beta|z|} \sin (\beta z)
\end{aligned}
$$

The radial displacement for the ramp-shaped fluid temperature profile was obtained by convolution integral as the following equation (Furuhashi et al., 2007, 2008).

$$
\begin{aligned}
& u(z)=R \alpha T_{f}(-\infty)+\int_{-\infty}^{\infty} \frac{d T_{f}(\xi)}{d \xi} G_{u}(z-\xi) d \xi=R \alpha T_{0}+\frac{\Delta T}{L} \int_{0}^{L} G_{u}(z-\xi) d \xi \\
& =R \alpha T_{m}(z)-\frac{m R \alpha \Delta T}{2 b L}\left(e^{-b|z|}-e^{-b|z-L|}\right)-\frac{m R \alpha \Delta T}{4 \beta L}\left\{\begin{array}{l}
e^{-\beta|z|} \sin (\beta|z|)-e^{-\beta|z|} \cos (\beta|z|) \\
-e^{-\beta|z-L|} \sin (\beta|z-L|) \\
+e^{-\beta|z-L|} \cos (\beta|z-L|)
\end{array}\right. \\
& +\frac{n R \alpha \Delta T}{2 \beta L}\left\{\begin{array}{l}
e^{-\beta|z|} \sin (\beta|z|)+e^{-\beta|z|} \cos (\beta|z|) \\
-e^{-\beta|z-L|} \sin (\beta|z-L|)-e^{-\beta|z-L|} \cos (\beta|z-L|)
\end{array}\right\}
\end{aligned}
$$


The thermal stresses were obtained as the following equations by substituting Eq.(34) into Eqs.(17),(18) and (19).

$$
\begin{aligned}
& S_{z b}(z)=\frac{\sigma_{z b}(z)}{E \alpha \Delta T}=\frac{\sqrt{3}}{\sqrt{1-v^{2}}} \frac{n}{b L}\left(e^{-b|z|}-e^{-b|z-L|}\right)+\frac{\sqrt{3}}{4 \sqrt{1-v^{2}}} \frac{m}{\beta L}\left\{\begin{array}{l}
e^{-\beta|z|} \sin (\beta|z|)+e^{-\beta|z|} \cos (\beta|z|) \\
-e^{-\beta|z-L|} \sin (\beta|z-L|) \\
-e^{-\beta|z-L|} \cos (\beta|z-L|)
\end{array}\right\} \\
& +\frac{\sqrt{3}}{2 \sqrt{1-v^{2}}} \frac{n}{\beta L}\left\{\begin{array}{l}
e^{-\beta|z|} \sin (\beta|z|)-e^{-\beta|z|} \cos (\beta|z|) \\
-e^{-\beta|z-L|} \sin (\beta|z-L|)+e^{-\beta|z-L|} \cos (\beta|z-L|)
\end{array}\right\} \\
& S_{h m}(z)=\frac{\sigma_{h m}(z)}{E \alpha \Delta T}=\frac{-m}{2 b L}\left(e^{-b|z|}-e^{-b|z-L|}\right)-\frac{m}{4 \beta L}\left\{\begin{array}{l}
e^{-\beta|z|} \sin (\beta|z|)-e^{-\beta|z|} \cos (\beta|z|) \\
-e^{-\beta|z-L|} \sin (\beta|z-L|)+e^{-\beta|z-L|} \cos (\beta|z-L|)
\end{array}\right\} \\
& +\frac{n}{2 \beta L}\left\{\begin{array}{l}
e^{-\beta|z|} \sin (\beta|z|)+e^{-\beta|z|} \cos (\beta|z|) \\
-e^{-\beta|z-L|} \sin (\beta|z-L|)-e^{-\beta|z-L|} \cos (\beta|z-L|)
\end{array}\right\} \\
& S_{h b}(z)=\frac{\sigma_{h b}(z)}{E \alpha \Delta T}=v S_{z b}(z)
\end{aligned}
$$

These solutions for the ramp-shaped fluid temperature profile, that are expressed by Eqs.(32) and (34) to (37), are symmetric with respect to the middle position, $z=L / 2$, and the middle temperature, $T_{0}+\Delta T / 2$. The $z$ terms in the right side express the effect of the lower end $(z=0)$ of stratified layer, and the $(z-L)$ terms express the effect of the upper end $(z=L)$ as well. The effects at both ends having opposite signs are paired and superposed, and decrease the stresses.

Since both $m$ and $n$ are functions of $b / \beta$ only, the non-dimensional stress $S$ exclusively depend on the ratio of coefficients, $b / \beta$, and the non-dimensional width of stratified layer, $\beta L$. When $L$ approaches 0 , the stresses approach the solutions for a step-shaped fluid temperature profile. Since one of the pair terms in the right side (either the $z$ term or the $(z-$ $L$ ) term) becomes negligibly small when $L$ approaches $\infty$, the stresses become inversely proportional to $L$. Practically you may consider $S \propto 1 / L$, when $b L>\pi$ and $\beta L>\Pi(L>2.5 \sqrt{R t})$.

When $t$ approaches $0(t=>0), b / \beta=>\infty, m=>1, n=>0$ and $T_{m}(z)=>T_{f}(z)$. In addition, when $\beta L>\pi(L>2.5 \sqrt{R t})$, thermal stresses become the maximum values at both ends of the stratified layer as expressed by the following equations.

$$
\begin{gathered}
S_{z b, \max }\left(\begin{array}{l}
z=0 \\
z=L
\end{array}\right)= \pm \frac{\sqrt{3}}{4 \beta L \sqrt{1-v^{2}}}, \quad \sigma_{z b, \max }\left(\begin{array}{l}
z=0 \\
z=L
\end{array}\right)= \pm \frac{3 E \alpha \sqrt{R t}}{4\left\{3\left(1-v^{2}\right)\right\}^{3 / 4}} \frac{\Delta T}{L} \\
S_{h m, \max }\left(\begin{array}{l}
z=0 \\
z=L
\end{array}\right)= \pm \frac{1}{4 \beta L}, \quad \sigma_{h m, \max }\left(\begin{array}{l}
z=0 \\
z=L
\end{array}\right)= \pm \frac{E \alpha \sqrt{R t}}{4 \sqrt[4]{3\left(1-v^{2}\right)}} \frac{\Delta T}{L}
\end{gathered}
$$

Eqs.(38) and (39) are well-known solutions (Timoshenko \& Woinowsky, 1959). They are often used as simple evaluation formulas in conventional structural design, because of the simplicity that the stress is proportional to the geometry parameter $(\sqrt{R t})$ and the 
temperature gradient $(\Delta T / L)$. However, it should be noted that these equations are applicable in the case where the vessel temperature corresponds to fluid temperature when $b / \beta=>\infty$, and $\beta L>\pi$.

\section{Verification by comparisons with FEM analysis}

\subsection{Step-shaped fluid temperature profile}

FEM analyses were performed for the case where the thermal stratification of the fluid (liquid sodium) contained in a reactor vessel of a large fast reactor occurs. The analysis conditions are as follows.

1. Applied FEM code: FINAS (CRC \& JAEA, 2006)

2. Reactor vessel: SUS316FR, $R=5350 \mathrm{~mm}, t=50 \mathrm{~mm}$

3. $T_{0}=350^{\circ} \mathrm{C}, \Delta \mathrm{T}=200^{\circ} \mathrm{C}$

4. Three cases for $L=0,4 t$ and $8 t$

5. Material characteristics are the values at the middle temperature of $450{ }^{\circ} \mathrm{C}$. $\lambda=21.512 \mathrm{~W} / \mathrm{mK}, E=164000 \mathrm{MPa}, \nu=0.301$ and $a=1.993 \times 10^{-5} /{ }^{\circ} \mathrm{C}$

6. Four cases for $h=3000,930,310$ and $100 \mathrm{~W} / \mathrm{m}^{2} \mathrm{~K}(B i=6.97,2.16,0.72,0.23)$

FEM results of the wall-averaged temperature for $L=0$ are shown in Fig. 4 with the note, (FEM), in the legend. The results calculated by the approximate solution based on the temperature profile method, Eq.(6), are also shown in Fig.4 with the note, (simple), in the legend. Both results agree well. FEM results of the thermal stress for $B i=2.16$ are shown in Fig.5 with the note, (FEM), in the legend. The results calculated by the shell solutions, Eqs.(23) to (25), are shown in Fig.5 with the note, (simple), in the legend. Both results agree well except the discrepancy of $S_{h b}$ for $z<3 t$. The discrepancy of $S_{h b}$ results from the assumption $\left(T_{b}=0\right)$ applied to the shell solution. Since FEM analysis is based on axisymmetric solid element, $T_{b}$ is naturally taken into account. It was confirmed that the shell solution obtained once again by substituting the binomial approximation of $T_{b}$ into Eqs.(14), (17) to (19) leads to good agreement with FEM analyses.

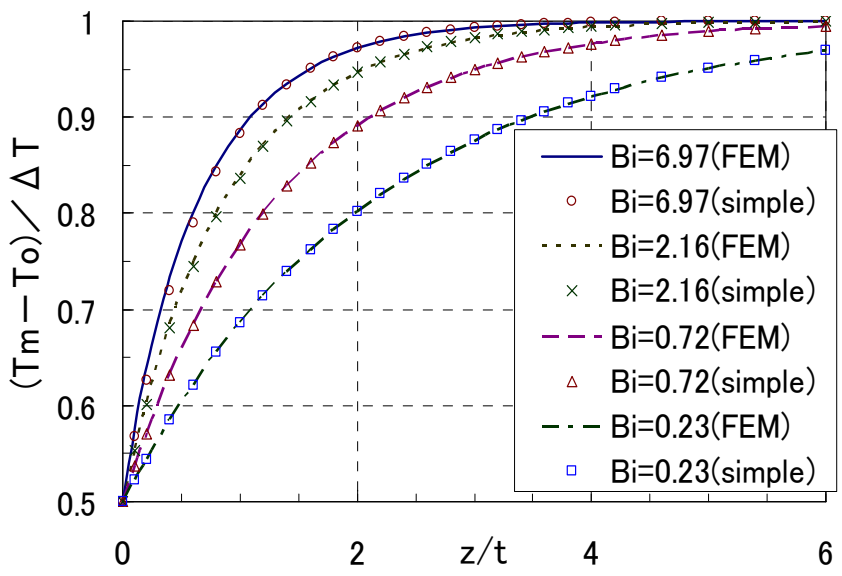

Fig. 4. Vessel temperatures for step-shaped fluid temperature 


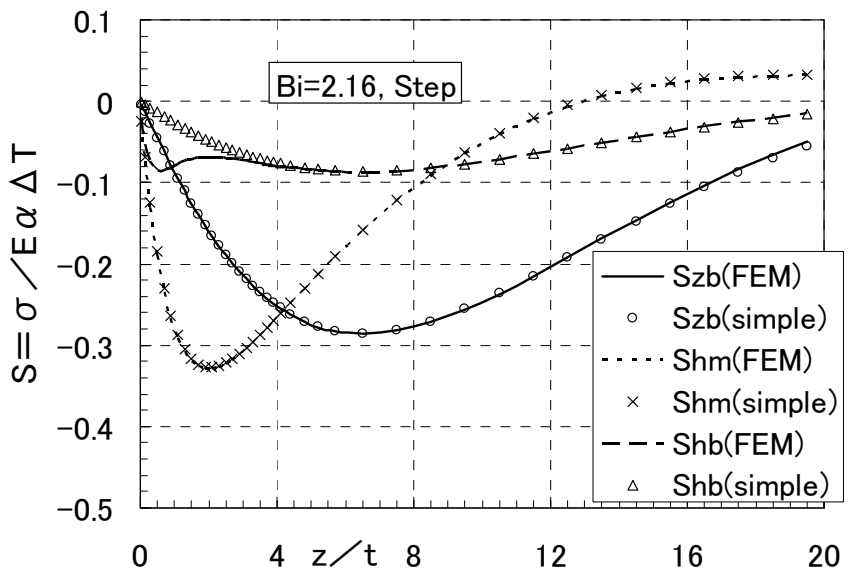

Fig. 5. Thermal stresses for step-shaped fluid temperature

However, it was decided that the shell solution with $T_{b}$ is not included in this paper, because (i) it doubles the number of terms in the stress solution, requiring too much space; and (ii) the discrepancy in $S_{h b}$ has minimal effect on the maximum stress evaluation. So we accepted the assumption $\left(T_{b}=0\right)$ in this paper.

The temperature and stress profiles are symmetric with respect to the middle position, $z=0$, and the middle temperature. Since this figure plots only the results in the hot section $(z>0)$, $S_{z b}$ and $S_{h m}$ shows the maximum negative (compressive) stress on the inner surface, respectively. In the cold section $(z<0)$, the maximum positive (tensile) stress occurs on the inner surface.

\subsection{Ramp-shaped fluid temperature profile}

Analyses were performed for the width $L=4 t$ and $8 t$, with $B i=6.97,2.16,0.72$ and 0.23 . Here, FEM results of the wall-averaged temperature for $L=8 t$ are shown in Fig. 6 with the note, (FEM), in the legend. The results calculated by the approximate solution, Eq.(32) are additionally shown with the note, (simple), in the legend. Both results agree well. FEM results of the thermal stress for $B i=2.16$ are shown in Fig.7 with the note, (FEM), in the legend. The results calculated by the shell solutions, Eqs.(35) to (37), are additionally shown with the note, (simple), in the legend. Both results agree well.

The temperature and stress profiles are symmetric with respect to the middle position, $z=L / 2$, and the middle temperature. Since this figure plots only the results in the hot section $(z>L / 2), S_{z b}$ and $S_{h m}$ shows the maximum negative (compressive) stress on the inner surface, respectively. In the cold section $(z<L / 2)$, the maximum positive (tensile) stress occurs on the inner surface.

\section{Simplified thermal stress evaluation chart}

The non-dimensional stress, $S$, exclusively depends on $b / \beta$ and $\beta L$. Using this characteristic, we developed simple charts to estimate maximum stress, $S$, and its generating location, $\Delta z$. And we proposed a simplified thermal stress evaluation method using these charts. The $S$ 


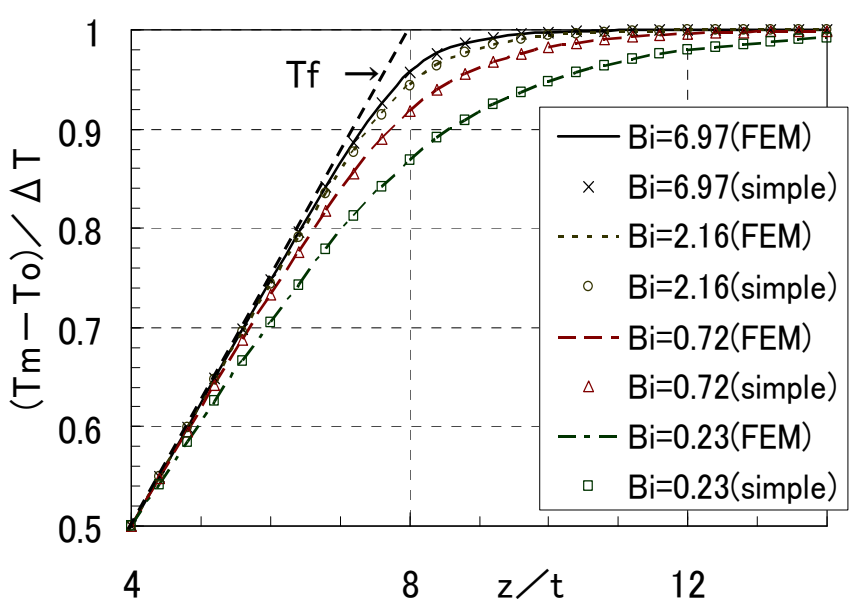

Fig. 6. Vessel temperatures for ramp-shaped fluid temperature

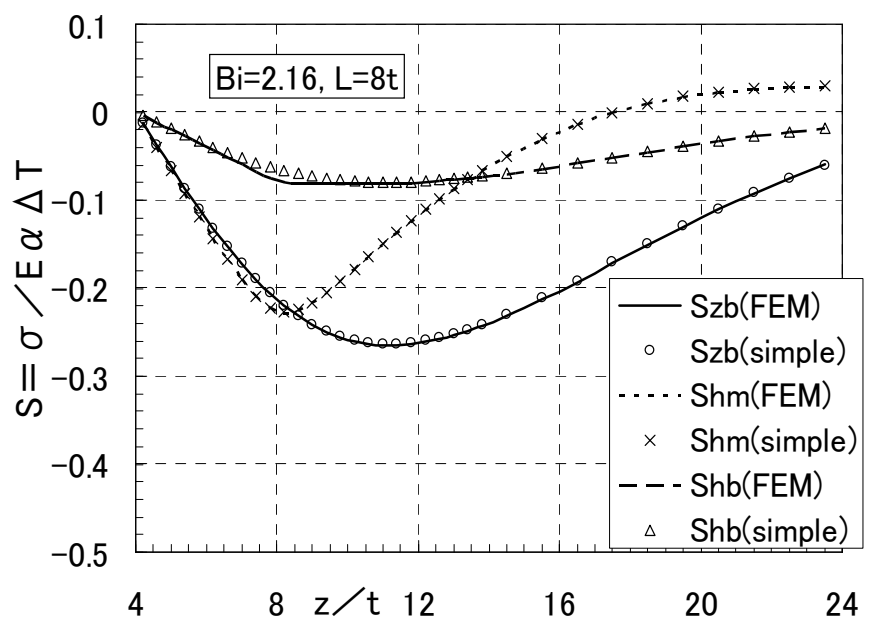

Fig. 7. Thermal stresses for ramp-shaped fluid temperature

charts were developed for $b / \beta>0.5$ and $\beta L<5$. When $b / \beta$ approaches $0, S$ approaches $0 . S$ is approximately inversely proportional to $L$ for $\beta L>5$. The maximum stress location, $\Delta z$, represents the outward distance from either end of the stratified layer. In the cold section, the maximum tensile (positive) stress occurs on the inner surface at $z=-\Delta z$, while in the hot section, the maximum compressive (negative) stress occurs on the inner surface at $z=L+\Delta z$. In addition, by substituting $z$ into Eq. (32), we can calculate the wall-averaged temperature, which is applicable to the reference temperature for material properties in structural design. 


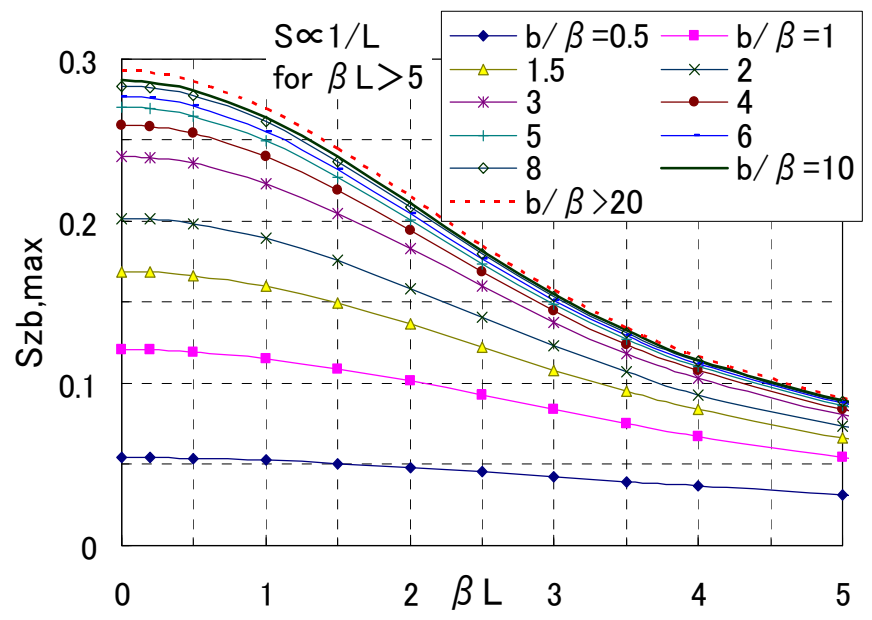

Fig. 8. The maximum bending stress

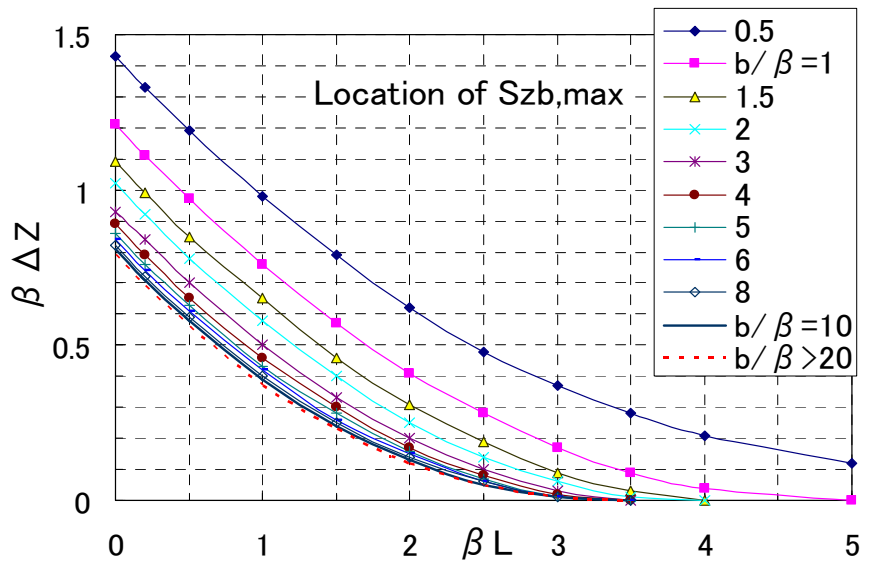

Fig. 9. Location of the maximum bending stress 


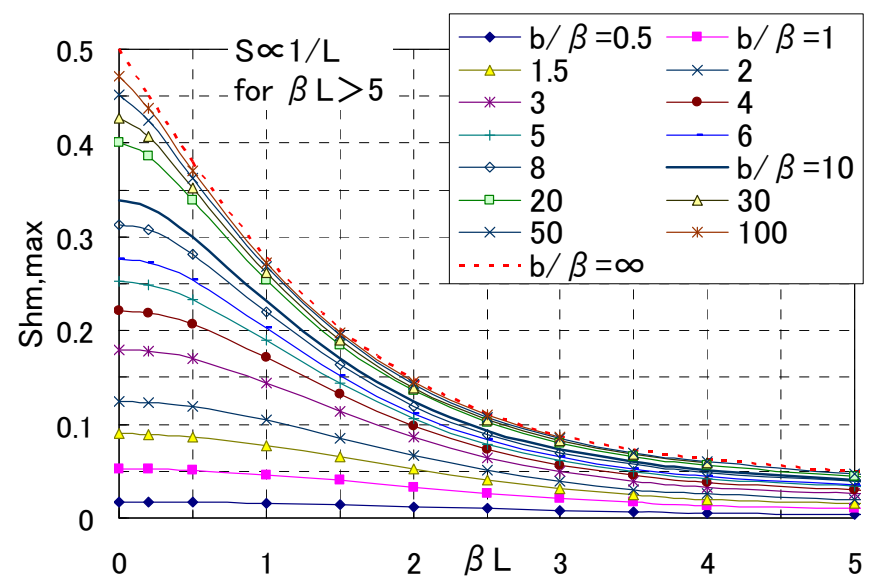

Fig. 10. The maximum membrane stress

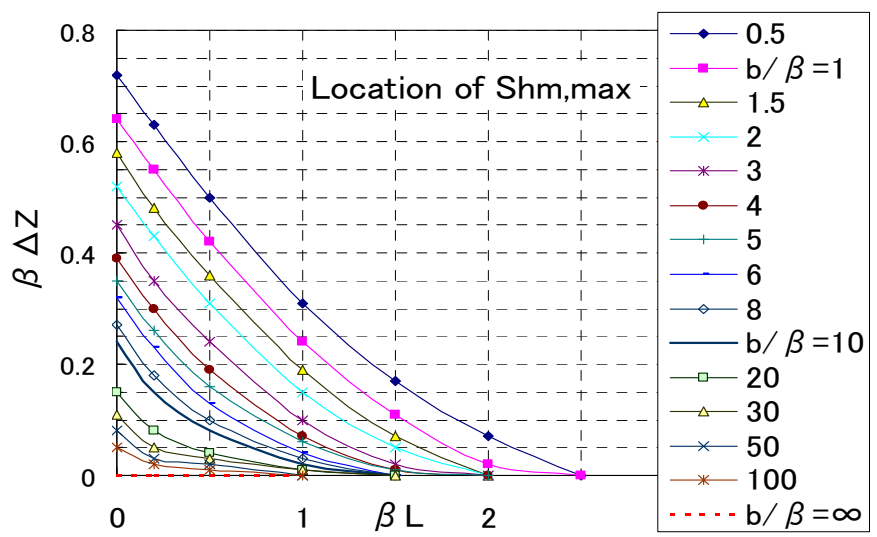

Fig. 11. Location of the maximum membrane stress 
The maximum bending stress, $S_{z b, \max }$ and its generating location, $\beta \Delta z$, is shown in Fig. 8 and Fig.9, respectively. The maximum membrane stress, $S_{h m, \max }$ and its generating location, $\beta \Delta z$, is shown in Fig.10 and Fig.11, respectively. The maximum stress intensity, $S_{n, \max }$ $\left(=\sigma_{S I, \max } / E a \Delta T\right)$ and its generating location, $\beta \Delta z$, is shown in Fig.12 and Fig.13, respectively. The stress intensity (Tresca's stress $\sigma_{S I}$ ) becomes the maximum value at the outer surface, where $\sigma_{z}$ and $\sigma_{h}$ have opposite signs.

$$
\sigma_{S I}=\operatorname{Max}\left(\left|\sigma_{z}\right|,\left|\sigma_{h}\right|,\left|\sigma_{z}-\sigma_{h}\right|\right)
$$

A small prominence observed in Fig.13 suggests the transition from the case that $S_{n, \max }$ occurs near the location of $S_{h m, \max }$ to the case that $S_{n, \max }$ occurs near the location of $S_{z b, \max }$.

The comparisons of FEM analyses, the proposed charts and the conventional method, Eq.(38) and (39), for 2 cases, $(L=8 t, B i=6.97)$ and $(L=4 t, B i=2.16)$, are shown in Table 1 . The parameters and $S$ values read out from the charts for the two cases are listed below.

$$
\begin{gathered}
\mathrm{L}, \mathrm{Bi}, \mathrm{b}, \beta, \mathrm{b} / \beta, \beta \mathrm{L}, \mathrm{S}_{\mathrm{zb}}, \mathrm{S}_{\mathrm{hm}}, \mathrm{S}_{\mathrm{n}} \\
\text { 8t, 6.97, 28.96, 2.48, 11.7, 0.99, } 0.27,0.24,0.39 \\
\text { 4t, 2.16, 22.41, 2.48, 9.03,0.50,0.28, } 0.30,0.43
\end{gathered}
$$

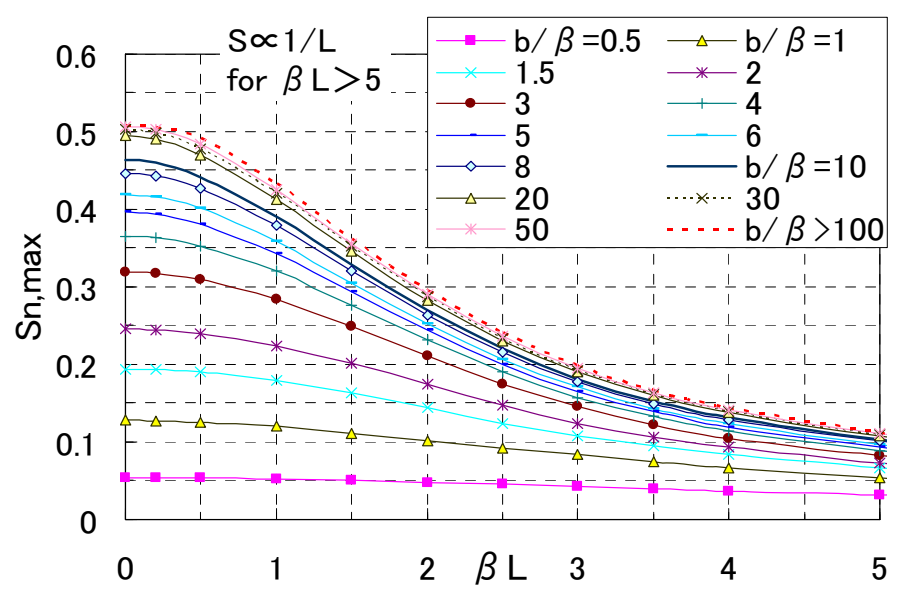

Fig. 12. The maximum stress intensity

It has been demonstrated that the proposed charts are sufficiently accurate. On the other hand, the conventional method leads to an overestimation. The main error is caused by the use of the formulas beyond the applicable range, $\beta L>\pi(L>2.5 \sqrt{R t})$. The comparison of the proposed method and the conventional method is shown in $S_{n}$-chart, Fig.14, and the above 2 cases results are plotted on the charts. 


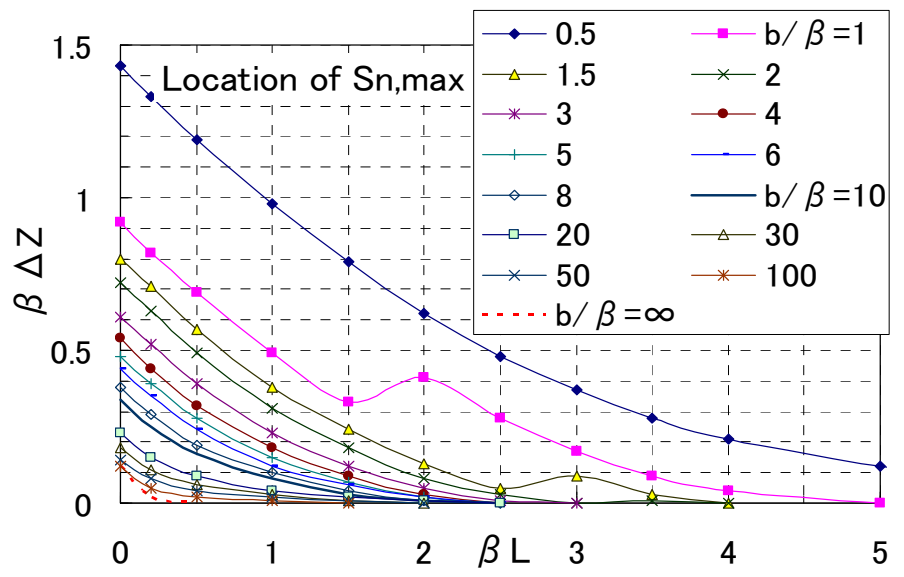

Fig. 13. Location of the maximum stress intensity

We often need to evaluate thermal stresses for observed thermal stratification phenomena in an engineering field. In most cases, axial temperature profile of interface between stratified fluid layers can be approximated by exponential curve or parabolic curve as shown in Fig.15 (Moriya et al., 1987; Haifeng et al., 2009; Kimura et al., 2010). We propose the effective width for such cases as following equation.

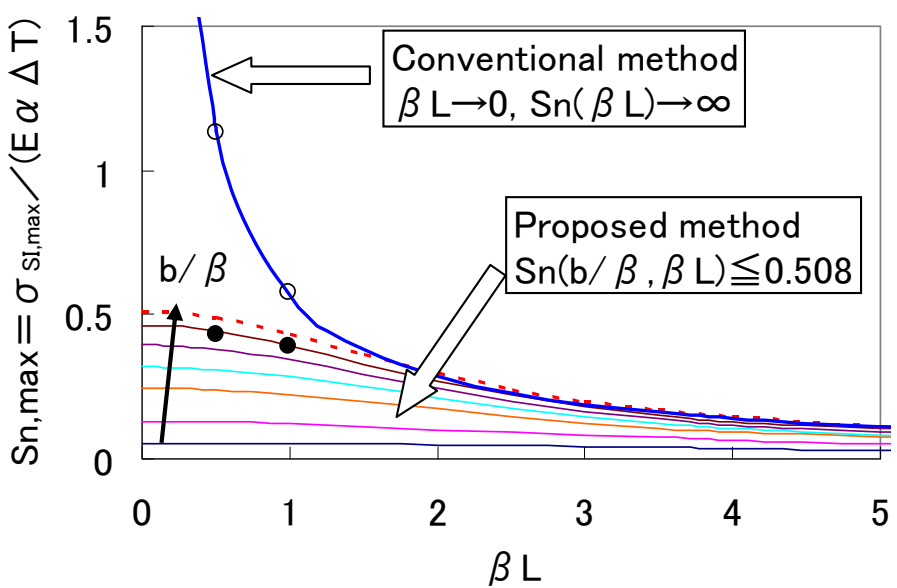

Fig. 14. Comparison of the proposed method and the conventional method 


\begin{tabular}{|c|c|c|c|c|c|c|c|}
\hline \multicolumn{2}{|c|}{ Method } & \multicolumn{2}{c|}{$\begin{array}{c}\text { Proposed } \\
\text { method }\end{array}$} & \multicolumn{2}{c|}{ FEM analyses } & \multicolumn{2}{c|}{$\begin{array}{c}\text { Conventional } \\
\text { method (38)(39) }\end{array}$} \\
\hline \multirow{2}{*}{ Case } & $\begin{array}{c}\text { Comp } \\
\text { onent }\end{array}$ & $\begin{array}{c}\sigma_{\max } \\
(\mathrm{MPa})\end{array}$ & $\begin{array}{c}\Delta z \\
(\mathrm{~mm})\end{array}$ & $\begin{array}{c}\sigma_{\max } \\
(\mathrm{MPa})\end{array}$ & $\begin{array}{c}\Delta z \\
(\mathrm{~mm})\end{array}$ & $\begin{array}{c}\sigma_{\max } \\
(\mathrm{MPa})\end{array}$ & $\begin{array}{c}\Delta z \\
(\mathrm{~mm})\end{array}$ \\
\hline \multirow{3}{*}{$\begin{array}{c}L=8 t \\
B i=6.97\end{array}$} & $S_{z b}$ & 177 & 149 & 175 & 150 & 300 & 0 \\
\cline { 2 - 9 } & $S_{h m}$ & 157 & 8 & 157 & 10 & 165 & 0 \\
\cline { 2 - 9 } & $S_{n}$ & 255 & 28 & 256 & 30 & 375 & 0 \\
\hline \multirow{3}{*}{$\begin{array}{c}\mathrm{B}=4 t \\
B i=2.16\end{array}$} & $S_{z b}$ & 184 & 234 & 183 & 225 & 600 & 0 \\
\cline { 2 - 9 } & $S_{h m}$ & 196 & 36 & 193 & 35 & 330 & 0 \\
\cline { 2 - 8 } & $S_{n}$ & 282 & 73 & 282 & 75 & 750 & 0 \\
\hline
\end{tabular}

Table 1. Comparison of stress evaluation results

$$
L_{e f f}=\frac{12}{\Delta T^{2}} \int_{T_{c}}^{T_{h}}\left(T_{f}-T_{m e d}\right) z d T_{f}
$$

$L_{\text {eff }}$ is nearly equal to the axial width corresponding to $90 \%$ of $\Delta T$ as shown in Fig.15. It is found that the thermal stress evaluations using the proposed charts and $L_{\text {eff }}$ are rather conservative and good evaluation, through comparisons with the FEM analyses.

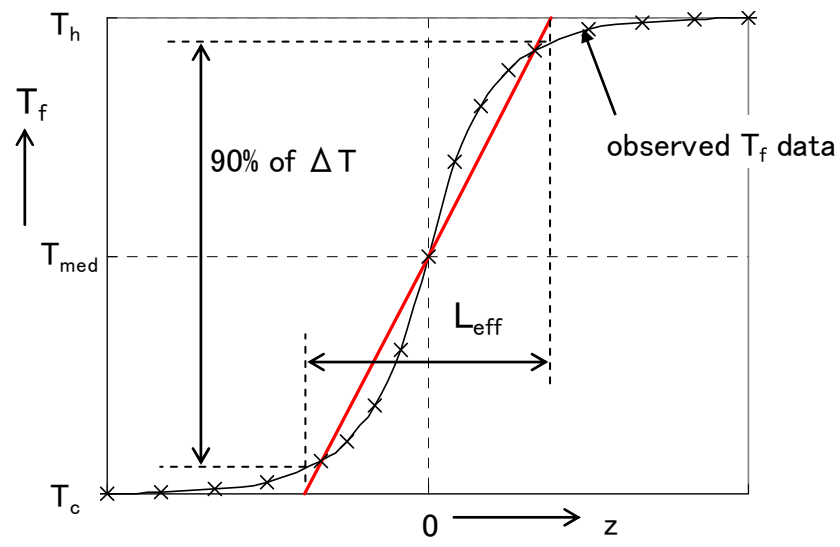

Fig. 15. Effective width of interface between stratified layers

\section{Conclusion}

To improve the accuracy of design evaluation methods of thermal stress induced by thermal stratification, this study have performed the theoretical analyses and FEM ones on steady-state temperature and thermal stress of cylindrical vessels, and obtained the following results.

1. The theoretical solution of steady-state temperature profiles of vessels and the approximate solution of the wall-averaged temperature based on the temperature profile method have been obtained. The wall-averaged temperature can be estimated with a high precision using the temperature attenuation coefficient, $b$. 
2. The shell theory solution for thermal stress based on the approximate solution of the wall-averaged temperature has been obtained. It has been demonstrated that the nondimensional thermal stress, $S=\sigma / E a \Delta T$ exclusively depends on the ratio of coefficients, $b / \beta$, and the non-dimensional interface width between stratified layers, $\beta L$.

3. Easy-to-use charts has been developed to estimate the maximum thermal stress and its generating location using the characteristic described in (2) above. In addition, a simplified thermal stress evaluation method has been proposed.

4. Through comparison with the FEM analysis results, it has been confirmed that the proposed method is sufficiently accurate to estimate the steady-state temperature and thermal stress.

5. It has been demonstrated that the conventional simple evaluation method using the shell stress solution, which assumes axial temperature profile consisting of a straight line with the maximum fluid temperature gradient, often leads to an overestimation.

6. For the convenient application of the proposed method to engineering problems, we proposed the effective width of interface between stratified layers. The thermal stress evaluation using the proposed charts with the effective width gives slightly conservative estimations.

The proposed method enables simple evaluations of steady-state thermal stress induced by thermal stratification taking the relaxation mechanism of thermal stress into account. This method would contribute to the reduction of design cost and to the rationalization of design.

\section{References}

Bieniussa, K.W. and Reck, H. (1996). Piping specific analysis of stresses due to thermal stratification, Nuclear Engineering and Design, Vol.190, No.1, pp. 239-249, ISSN:00295493.

Carslaw, H.S. and Jeager, J.C. (1959). Conduction of heat in solids, 2nd edition, pp. 166-169, Oxford University Press.

CRC Solutions Corp. \& Japan Atomic Energy Agency (2006). FINAS User's Manual version 18.0, (in Japanese).

Furuhashi, I., Kawasaki, N. and Kasahara, N. (2007). Evaluation Charts of Thermal Stresses in Cylindrical Vessels Induced by Thermal Stratification of Contained Fluid, (in Japanese), Transactions of the Japan Society of Mechanical Engineers, Series A, Vol.73, No.730, pp. 686-693.

Furuhashi, I., Kawasaki, N. and Kasahara, N. (2008). Evaluation Charts of Thermal Stresses in Cylindrical Vessels Induced by Thermal Stratification of Contained Fluid, Journal of Computational Science and Technology, Vol.2, No.4, pp. 547-558.

Furuhashi, I. and Watashi, K. (1991). A Simplified Method of Stress Calculation of a Nozzle Subjected to a Thermal Transient, International Journal of Pressure Vessels and Piping, Vol.45, pp. 133-162, ISSN:0308-0161.

Haifeng, G. et al. (2009). Experimental Study on the Fluid Stratification Mechanism in the Density Lock, Journal of NUCLEAR SCIENCE and TECHNOLOGY, Vol.46, No.9, pp. 925- 932, ISSN:0022-3131

Kimura, N. et al. (2010). Experimental Study on Thermal Stratification in a Reactor Vessel of Innovatic Sodium-Cooled Fast Reactor - Mitigation Approach of Temperature Gradient across Stratification Interface -, Journal of NUCLEAR SCIENCE and TECHNOLOGY, Vol.47, No.9, pp. 829- 838, ISSN:0022-3131 
Katto, Y. (1964), Conduction of Heat, (in Japanese), (1964), p.38, Yokendo.

Moriya, S. et al. (1987). Effects of Reynolds Number and Richardson Number on Thermal Stratification in Hot Plenum, Nuclear Engineering and Design, Vol.99, pp. 441-451, ISSN:0029-5493.

Morse, P.M. and Feshbach, H. (1953). Methods of Theoretical Physics, Part.1, pp. 710-730, McGraw-Hill.

Timoshenko, S.P. and Woinowsky-Krieger, S. (1959). Theory of plates and shells, 2nd edition, pp. 466-501, McGraw-Hill. 


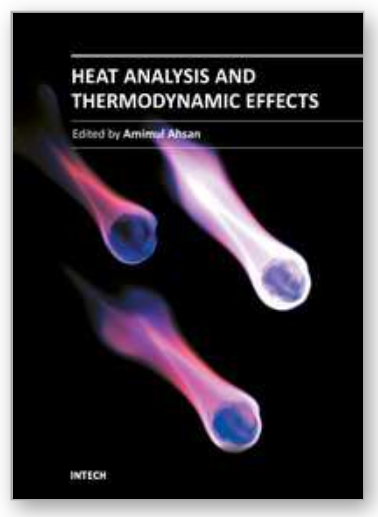

\author{
Heat Analysis and Thermodynamic Effects \\ Edited by Dr. Amimul Ahsan
}

ISBN 978-953-307-585-3

Hard cover, 394 pages

Publisher InTech

Published online 22, September, 2011

Published in print edition September, 2011

The heat transfer and analysis on heat pipe and exchanger, and thermal stress are significant issues in a design of wide range of industrial processes and devices. This book includes 17 advanced and revised contributions, and it covers mainly (1) thermodynamic effects and thermal stress, (2) heat pipe and exchanger, (3) gas flow and oxidation, and (4) heat analysis. The first section introduces spontaneous heat flow, thermodynamic effect of groundwater, stress on vertical cylindrical vessel, transient temperature fields, principles of thermoelectric conversion, and transformer performances. The second section covers thermosyphon heat pipe, shell and tube heat exchangers, heat transfer in bundles of transversely-finned tubes, fired heaters for petroleum refineries, and heat exchangers of irreversible power cycles. The third section includes gas flow over a cylinder, gas-solid flow applications, oxidation exposure, effects of buoyancy, and application of energy and thermal performance index on energy efficiency. The forth section presents integral transform and green function methods, micro capillary pumped loop, influence of polyisobutylene additions, synthesis of novel materials, and materials for electromagnetic launchers. The advanced ideas and information described here will be fruitful for the readers to find a sustainable solution in an industrialized society.

\title{
How to reference
}

In order to correctly reference this scholarly work, feel free to copy and paste the following:

Ichiro Furuhashi (2011). Stress of Vertical Cylindrical Vessel for Thermal Stratification of Contained Fluid, Heat Analysis and Thermodynamic Effects, Dr. Amimul Ahsan (Ed.), ISBN: 978-953-307-585-3, InTech, Available from: http://www.intechopen.com/books/heat-analysis-and-thermodynamic-effects/stress-of-vertical-cylindricalvessel-for-thermal-stratification-of-contained-fluid

\section{INTECH}

open science | open minds

\author{
InTech Europe \\ University Campus STeP Ri \\ Slavka Krautzeka 83/A \\ 51000 Rijeka, Croatia \\ Phone: +385 (51) 770447 \\ Fax: +385 (51) 686166 \\ www.intechopen.com
}

\author{
InTech China \\ Unit 405, Office Block, Hotel Equatorial Shanghai \\ No.65, Yan An Road (West), Shanghai, 200040, China \\ 中国上海市延安西路65号上海国际贵都大饭店办公楼 405 单元 \\ Phone: +86-21-62489820 \\ Fax: +86-21-62489821
}


(C) 2011 The Author(s). Licensee IntechOpen. This chapter is distributed under the terms of the Creative Commons Attribution-NonCommercialShareAlike-3.0 License, which permits use, distribution and reproduction for non-commercial purposes, provided the original is properly cited and derivative works building on this content are distributed under the same license. 